\title{
Hiding Information in 3D Printed Objects by Forming Fine Cavities inside Objects
}

\author{
Kazutake Uehira ${ }^{1}$, Satoru Baba ${ }^{2}$, Masahiro Suzuki ${ }^{3}$, Piyarat Silapasuphakornwong ${ }^{4}$, Hideyuki Torii ${ }^{5}$, \\ Youichi Takashima ${ }^{6}$ \\ ${ }^{1-5}$ Kanagawa Institute of Technology \\ 1030 Shimo-ogino, Atsugi, Japan \\ uehira@nw.kanagawa-it.ac.jp; msuzuki@ctr.kanagawa-it.ac.jp; silpiyarat@gmail.com; torii@nw.kanagawa-it.ac.jp \\ ${ }^{6}$ NTT Service Evolution Laboratories/ Nippon Telegraph and Telephone Corporation \\ 1-1 Hikarinoka, Yokosuka, Japan \\ takashima.youichi@lab.ntt.co.jp
}

\section{Extended Abstract}

This paper presents a copyright protection technique for 3D printing by hiding information inside 3D-printed objects. In the future when 3D printers become widespread, people will purchase the digital data of objects they want to produce from Web sites, download it, and manufacture objects at home with 3D printers instead of purchasing real objects. In this situation, the problem of illegal copies of digital data will become serious because digital data is easy to copy. Therefore, copyright protection for digital data for 3D printing will become important.

We previously proposed a technique that can protect the copyrights of digital data for products manufactured by 3D printers [1]. The basic concept of our technique is as follows. When the providers prepare the contents of a 3D object using the 3D-CAD, the copyright information is integrated to the object data. After that, when a customer purchases the digital data through the internet and prints out 3D objects by using a household 3D printer, the fine cavities are simultaneously formed inside the physical 3D object by integrating copyright information during its fabrication. The disposition of the fine cavities expresses the information, that is, existence or non-existence of a cavity in a designated position inside the object expresses binary data, " 1 " or " 0 ". We also proposed a technique that can non-destructively read out information from inside the objects using thermography. If we form the cavity near the surface of the object and raise the temperature of the surface by heating, the surface regions under which cavities exist become warmer than the other regions because of the low-thermal conductivity of the cavity. Therefore, we can find out the disposition of the fine cavities from the thermal image captured with thermography, that is, we can read out embedded information. In a previous study, we just showed the feasibility using a sample with flat and spherical surfaces [1], [2].

In this paper, we study the readability of embedded information relating with the structure parameters of the fine cavities inside real objects and try to clarify the conditions in which this technique can be applied. We conducted an experiment using samples fabricated by a stereolithographic 3D printer, and polylactide (PLA) resin was used as the material for the sample, which was $5 \times 5 \times 1 \mathrm{~cm}$. The size of the cavities, the space between cavities, and the depth of the cavities from the surface of the object were changed as experimental parameters.

Experimental results reveal that a cavity of at least $1.5 \mathrm{x} 1.5 \mathrm{~mm}$ can be detected. This size is the top viewed size, and all cavities are $2 \mathrm{~mm}$ high. It is also seen that cavities over $1.5 \mathrm{~mm}$ apart can be detected separately. Moreover, the cavities at the depth of within $2 \mathrm{~mm}$ from the surface can be detected.

From these results, the conditions for forming cavities inside the fabricated object are clarified. Moreover, these results show that a sufficient amount of information for copyright (i.e. hundreds bits) can be embedded if a fabricated object is several centimetres in size.

\section{References}

[1] M. Suzuki, P. Silapasuphakornwong, K. Uehira, et al., "Copyright Protection for 3D Printing by Embedding Information Inside Real Fabricated Objects,” in Proc. VISIGRAPP 2015, 2015, pp. 1-6. 
[2] A. Okada, P. Silapasuphakornwong, K. Uehira, et al., "Non-destructively reading out information embedded inside real objects by using far-infrared light," in Proc. of 2015 SPIE OPTICS+PHOTONICS, 2015, pp. 9599-106. 